\title{
A Ray-Tracing Model of the Vela Magnetosphere
}

\author{
C. Hirano and C. R. Gwinn \\ Department of Physics, University of California, Santa Barbara, CA \\ 93106
}

\begin{abstract}
In the relativistic plasma surrounding a pulsar, a subluminal ordinary-mode electromagnetic wave will propagate along a magnetic field line. After some distance, it can break free of the field line and escape the magnetosphere to reach an observer. We apply a simple model of pulsar radio emission based on this scenario to the case of the Vela pulsar and find it reproduces some qualitative characteristics of the observed Vela pulse profile.
\end{abstract}

\section{Wave Propagation in the Pulsar Magnetosphere}

Barnard \& Arons (1986) investigated the propagation of electromagnetic waves in the relativistic plasma permeating the pulsar magnetosphere and found that one mode, the subluminal $\mathrm{O}$-mode, would follow paths along the magnetic field lines with the direction of the wave vector $\mathbf{k}$ approximately conserved. If some mechanism converts the subluminal mode to the superluminal mode when the two modes are most alike, the ray can break free of the plasma and reach an observer. Observations of rays originating in the subluminal mode would therefore reflect the geometry of the dipolar field (Gallant 1996), which could account for some observed pulse shapes and position angle sweeps.

\section{Results}

Figure 1 illustrates the paths of several rays that reach an observer. The generated pulse, shown in Figure 2 consists of a strong pulse followed by a weaker subpulse about one fifth to one fourth as strong, like the observed pulse from Vela. Also, the average position angle sweeps through roughly the same range as is observed.

\section{References}

Barnard, J. J., \& Arons, J. 1986, ApJ, 302, 138

Gallant, Y. A. 1996, in IAU Colloq. 160, Pulsars: Problems and Progress, ed. S. Johnston, M. A. Walker, and M. Bailes (San Francisco: Astronomical Society of the Pacific), 431 


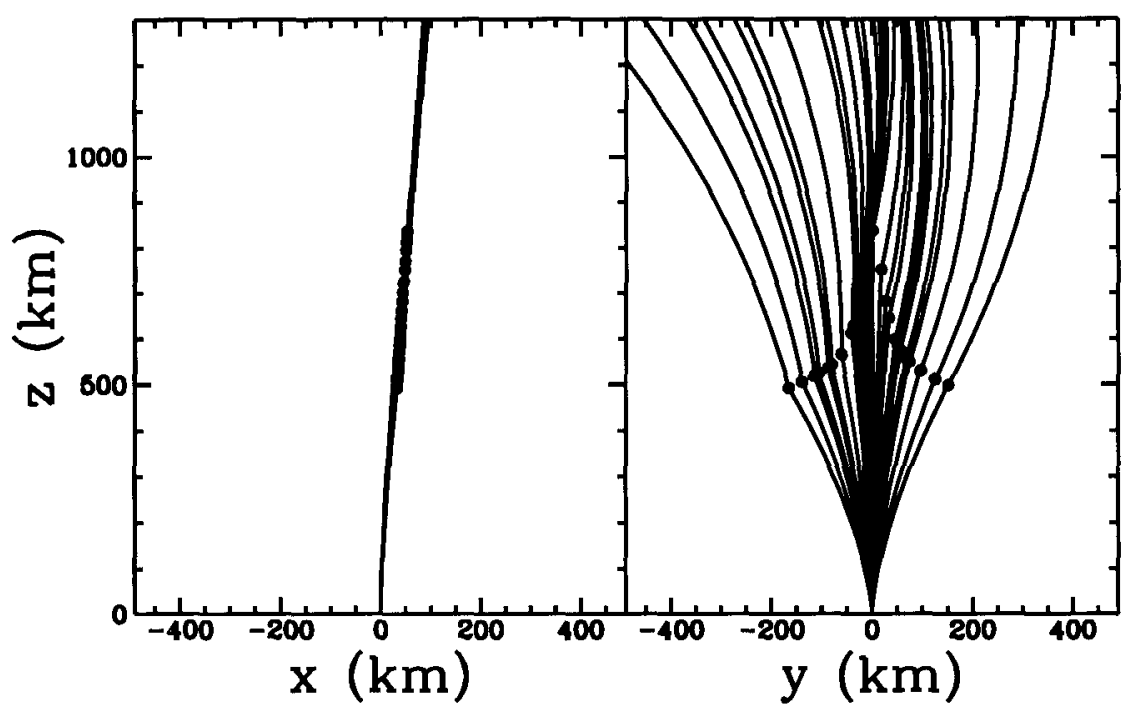

Figure 1. The paths of several rays, as seen in the corotating frame, projected onto the $x z$ - and $y z$-planes. The magnetic axis lies along the $z$-axis, and the rotation axis lies in the $x z$-plane. Until the mode transition, the rays follow the magnetic field lines; after the transition, they trace Archimedean spirals as seen from the corotating frame.
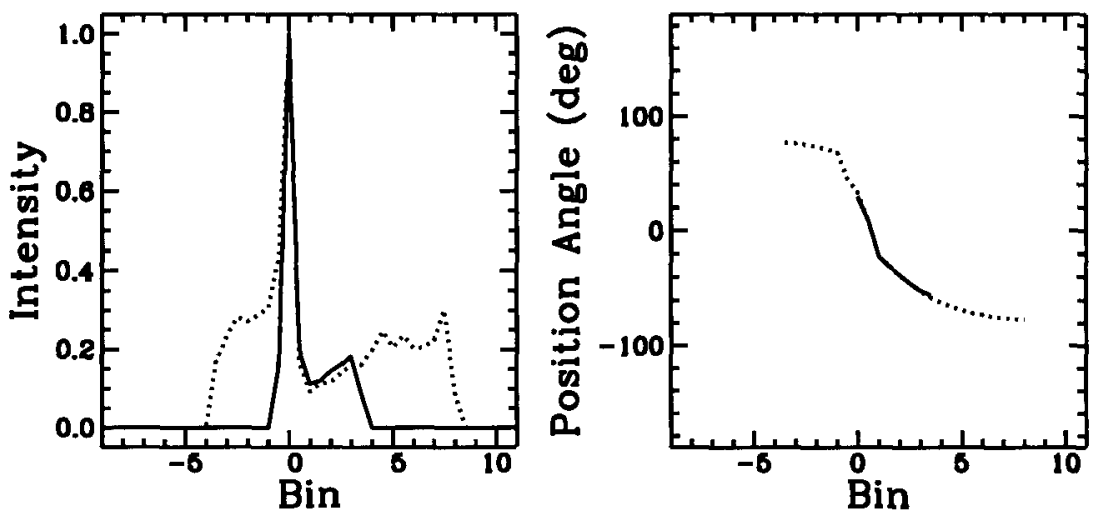

Figure 2. Model-generated pulse profiles at $2.3 \mathrm{GHz}$. The pulse period consists of one hundred bins. Limiting the size of the emission area on the stellar surface results in a considerably narrower pulse, shown in solid, providing better agreement with observation. 